\title{
Coherent Jetting from a Gate-Defined Channel in Bilayer Graphene
}

\author{
Carolin Gold๑, ${ }^{1,}$ Angelika Knothe, ${ }^{2}$ Annika Kurzmann, ${ }^{1}$ Aitor Garcia-Ruiz, ${ }^{2}$ Kenji Watanabe $\odot,{ }^{3}$ Takashi Taniguchi, ${ }^{4}$ \\ Vladimir Fal'ko, ${ }^{2,5,6}$ Klaus Ensslin $\oplus^{1,7}$ and Thomas Ihn $\oplus^{1,7}$ \\ ${ }^{1}$ Solid State Physics Laboratory, ETH Zürich, CH-8093 Zürich, Switzerland \\ ${ }^{2}$ National Graphene Institute, University of Manchester, Manchester M13 9PL, United Kingdom \\ ${ }^{3}$ Research Center for Functional Materials, National Institute for Materials Science, 1-1 Namiki, Tsukuba 305-0044, Japan \\ ${ }^{4}$ International Center for Materials Nanoarchitectonics, National Institute for Materials Science, 1-1 Namiki, Tsukuba 305-0044, Japan \\ ${ }^{5}$ Department of Physics, University of Manchester, Manchester M13 9PL, United Kingdom \\ ${ }^{6}$ Henry Royce Institute, University of Manchester, Manchester M13 9PL, United Kingdom \\ ${ }^{7}$ Quantum Center, ETH Zürich, 8093 Zürich, Switzerland
}

(Received 19 November 2020; accepted 18 May 2021; published 20 July 2021)

\begin{abstract}
Graphene has evolved as a platform for quantum transport that can compete with the best and cleanest semiconductor systems. Here, we report on the observation of distinct electronic jets emanating from a narrow split-gate-defined channel in bilayer graphene. We find that these jets, which are visible via their interference patterns, occur predominantly with an angle of $60^{\circ}$ between each other. This observation is related to the trigonal warping in the band structure of bilayer graphene, which, in conjunction with electron injection through a constriction, leads to a valley-dependent selection of momenta. This experimental observation of electron jetting has consequences for carrier transport in two-dimensional materials with a trigonally warped band structure in general, as well as for devices relying on ballistic and valley-selective transport.
\end{abstract}

DOI: 10.1103/PhysRevLett.127.046801

The remarkable electronic properties of graphene-based van der Waals heterostructures has led to a broad interest in both their possible applications, as well as fundamentally new physics phenomena $[1,2]$. The possibility to electrically control the band gap in an unprecedented manner [3-5] makes bilayer graphene a promising platform for fundamental quantum-electronic building blocks [6-9]. Within this context, the valley degree of freedom and its possible application in valleytronic devices are a key paradigm for both classical and quantum information processing and computing $[10,11]$. Valley-polarized electron jets have been predicted [12-14] to occur in monolayer graphene channels of certain orientation, if the dispersion at the Fermi energy in the bulk is trigonally warped. However, the experimental realization of such devices is challenging, as the observation of electron jetting requires (i) an experimentally accessible trigonally warped dispersion, (ii) a tunable, electrostatically defined electronic constriction avoiding edge disorder, and (iii) a clean bulk allowing for ballistic transport over micrometer distances.

Only recently, technological advances [6-9] enabled to utilize the unique properties of bilayer graphene for

Published by the American Physical Society under the terms of the Creative Commons Attribution 4.0 International license. Further distribution of this work must maintain attribution to the author(s) and the published article's title, journal citation, and DOI. observing this effect. Electron flow through these devices can be imaged with scanning gate microscopy (SGM). This technique was used to image a plethora of local phenomena [15-20], one milestone experiment being the observation of branched electron flow in the two-dimensional electron gas behind a quantum point contact in $\mathrm{Ga}(\mathrm{Al})$ As heterostructures [21-23].

Here, we use SGM to explore the modification of electronic transport in bilayer graphene in close vicinity to a gate-defined constriction. The resulting conductance maps depict two distinct jets, which emanate from the constriction with a $60^{\circ}$ angle between them. The angular distribution of the jets is a result of the preferential selection of momenta due to the lowest-energy modes of the constriction and the trigonally warped bulk dispersion of the gapped bilayer graphene relevant for ballistic electron motion. It is stable against variations of the channel orientation with respect to the graphene edge, except for angles of $30^{\circ}$. The origin of the jets is in stark contrast to the small-angle scattering origin of electron branching observed in similar measurements on $\mathrm{Ga}(\mathrm{Al}) \mathrm{As}$ heterostructures [22,23]. Due to its origin, the electron jetting along preferred crystallographic directions observed in our measurements is of general relevance for carrier transport in two-dimensional materials with a trigonally warped band structure (including bi- and multilayer graphene [24], $\mathrm{MoS}_{2}$ [25], and other transition metal dichalcogenides) and opens new avenues for the design of quantum devices. 
Experiment.-Our measurement setup consists of a nanostructured van der Waals stack and a metallic SGM-tip scanning above it, as depicted schematically in Fig. 1(a). The stack consists of a bilayer graphene (BLG) flake encapsulated between 32(40) nm thick hexagonal boron nitride ( $h$-BN). Fabricated as in Ref. [6], the BLG is contacted by source and drain contacts and gated by a graphite back gate (BG) and two metallic split gates (SGs) on top of the device (300 $\mathrm{nm}$ long and $50 \mathrm{~nm}$ apart). The joint action of the voltage $V_{\mathrm{SG}}$ applied to both split gates and the back-gate voltage $V_{\mathrm{BG}}$ results in a gate-induced displacement field $D=\left[C_{\mathrm{BG}}\left(V_{\mathrm{BG}}-V_{\mathrm{BG}}^{0}\right)-C_{\mathrm{SG}}\left(V_{\mathrm{SG}}-V_{\mathrm{SG}}^{0}\right)\right] /\left(2 \epsilon_{0}\right)$ in the split-gated area. Here, $C_{\mathrm{SG}}\left(C_{\mathrm{BG}}\right)$ are the split(back)-gate capacitances per unit area [26] and $V_{\mathrm{SG}}^{0}\left(V_{\mathrm{BG}}^{0}\right)$ are the charge neutrality point offsets from zero in the double-gated regions. For appropriate gate voltages, the resulting displacement field opens a band gap in the double-gated region while simultaneously keeping the Fermi energy in the gap. The BLG below the split gates is thus insulating and the source-drain current is constrained to the noninsulating channel between them (see Ref. [3] for details).

To study the spatial structure of electron flow in the graphene bulk behind the channel at $T=270 \mathrm{mK}$, we raster scan the voltage-biased, metallic SGM tip $30 \mathrm{~nm}$ above the surface of the top $h$-BN. Applying an ac source-drain voltage $V_{\mathrm{SD}}=50 \mu \mathrm{V}$, we measure the linear conductance $G(x, y)=I_{\mathrm{SD}}(x, y) / V_{\mathrm{SD}}$ as a function of the tip position $(x, y)$ in the areas behind the channel [cf. red-marked areas in Fig. 1(a)]. The tip capacitively influences the charge density and the energy gap of the BLG locally, thus scattering electrons. Unless stated otherwise, all our measurements are performed at the tip voltage $V_{\text {tip }}=-10 \mathrm{~V}$, which we expect to induce a depletion region below the tip (see Supplemental Material [28]).

Channel formation.-To characterize the interplay of the split- and back-gate voltages, we measure the conductance $G\left(V_{\mathrm{SG}}, V_{\mathrm{BG}}\right)$ shown in Fig. 1(b) in the absence of the tip. The two split-gate-independent conductance minima at finite back-gate voltage arise when the Fermi energy in the regions outside the split-gated area lies at the charge neutrality point [34]. The third conductance minimum depends linearly on both the split-gate and the back-gate voltage [cf. diagonal, partly red-marked line of reduced conductance in Fig. 1(b)]. Along this minimum, the gate-induced displacement field $D$ renders the split-gated regions insulating, thus confining the source-drain current to the remaining conductive region between the gates. As the displacement field increases for larger gate-voltage differences, the band gap increases along the minimum in the direction of the red arrow in Fig. 1(b).

Because the electronic channel couples to the split gates via stray fields only, the electrostatic coupling of the channel to the gates is dominated by the back gate. The electron density in the channel thus increases with increasing displacement field, and the pinch-off of the channel observed for $D \in[0.21,0.35] \mathrm{V} / \mathrm{nm}$ suggests that the
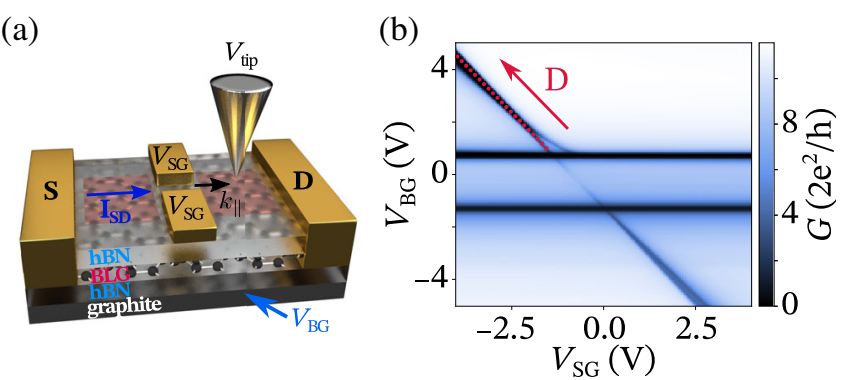

(c)

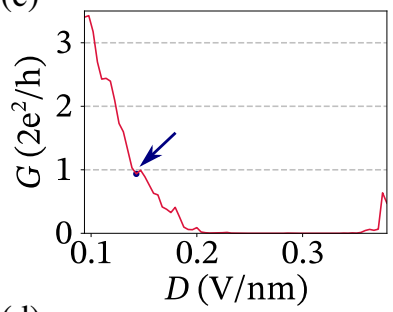

(e)

(f)

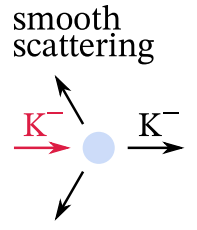

atomic-scale scattering

(d)

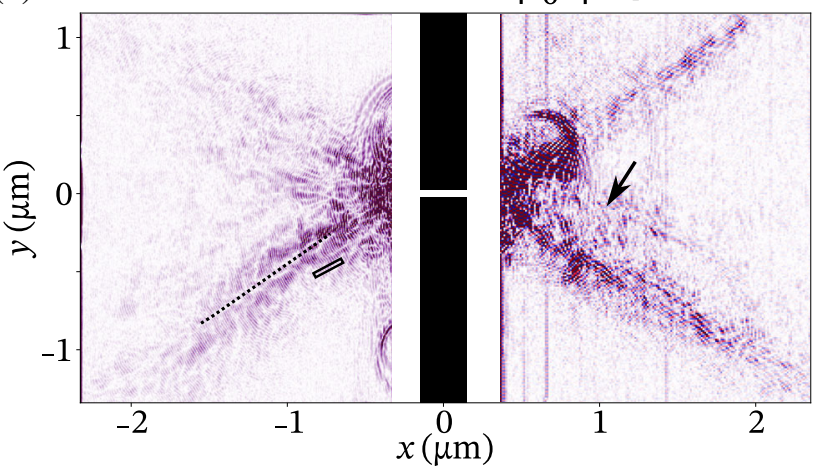

FIG. 1. (a) Measurement setup schematic. The metallic tip is scanned above the sample in the areas denoted by the red rectangles. (b) Conductance $G\left(V_{\mathrm{SG}}, V_{\mathrm{BG}}\right)$. Charge neutrality (CNP) underneath the split gates and hence the formation of a channel is achieved along the diagonal conductance minimum. These data have also been published in a previous work [27]. (c) Conductance as a function of the displacement field $D$ along the CNP (cf. red dotted line). (d) Filtered conductance $G_{f}(x, y)$ [28] on both sides of the channel for $V_{\text {tip }}=-10 \mathrm{~V}$. Clear jets with interference patterns emanate from the channel predominantly with a $60^{\circ}$ angle between them. The black arrow marks an example of a secondary jet. Because of rapid oscillations in the data, the color scale appears purple. (e),(f) Basic scattering processes possible in the sample. (e) Valley-conserving scattering off a smooth scattering potential. (f) Valley-conserving (black arrows) and valley-altering (orange arrows) atomic-scale scattering.

channel reaches its charge neutrality point in this range of displacement fields [27].

Along the red-dotted line in Fig. 1(b), the conductance through the channel varies with displacement field as depicted in Fig. 1(c). While the current is completely suppressed at the aforementioned displacement fields, the conductance increases for decreasing displacement field. Since the channel's charge neutrality point is within $D \in[0.21,0.35] \mathrm{V} / \mathrm{nm}$, the Fermi energy for $D<0.21 \mathrm{~V} / \mathrm{nm}$ lies close to or even within the valence 
band within the channel. This renders the channel $p$ type, whereas the bulk around the channel as well as the channel ends are $n$ type. All our SGM measurements are performed at a displacement field $D \approx 0.14 \mathrm{~V} / \mathrm{nm}$, for which the conductance is approximately $2 e^{2} / h$ [cf. blue arrow and point in Fig. 1(c)].

SGM behind the channel. - We perform SGM measurements in the regions indicated by red squares on both sides of the split gates in Fig. 1(a). The filtered conductance $G_{f}$ of the resulting conductance map is depicted in Fig. 1(d). The filtering procedure (see Supplemental Material [28]) removes the background and eliminates high-frequency noise on length scales smaller than half the Fermi wavelength. On both sides of the channel, the filtered conductance $G_{f}$ exhibits narrow jets with interference fringes emanating from the channel predominantly at an angle of $60^{\circ}$ with respect to each other. In proximity to the main jets, we observe secondary jets as, e.g., indicated by the black arrow in Fig. 1(d). In contrast to branched-flow measurements on $\mathrm{Ga}(\mathrm{Al}) \mathrm{As}$ heterostructures [21,22], we observe no significant reduction of the conductance along the jets. Measurements at small, perpendicularly applied magnetic fields (see Supplemental Material [28]) reveal that the strongest interferences are observed for radial distances from the channel of the order of the cyclotron orbit. This indicates the relation of the jets to ballistic electron transport. Furthermore, the jets are stable against small variations in the experimental parameters (see Supplemental Material [28]).

To study the influence of the tip voltage on the interference fringes, we measure the conductance $G\left(x, V_{\text {tip }}\right)$ for tip positions $x$ along the black dotted line in Fig. 1(d). The numerical derivative $d G\left(x, V_{\text {tip }}\right) / d x$ is depicted in Fig. 2(a). We observe interference fringes for $V_{\text {tip }}<-4 \mathrm{~V}$ far below the least-invasive tip voltage $V_{\text {tip }}^{\mathrm{li}} \approx 0.25 \mathrm{~V}$ (see [28]). These interference patterns almost
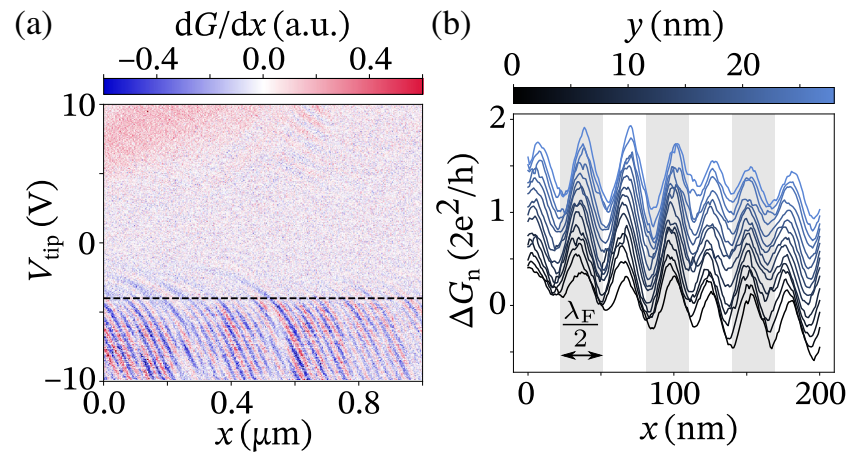

FIG. 2. (a) Numerical derivative of the conductance $G\left(x, V_{\text {tip }}\right)$ along the black dotted line in Fig. 1(d). Interferences are observed below the black-dashed guide to the eye. (b) Normalized conductance $\Delta G_{n}(x, y)$ [28] measured within the black rectangle in Fig. 1(d). Each line is vertically offset with respect to the previous line. A periodicity of $\lambda_{F} / 2 \approx 29 \mathrm{~nm}$ is observed (cf. black arrow). completely disappear at $V_{\text {tip }} \geq-4 \mathrm{~V}$ [cf. above the dashed black line in Fig. 2(a)] and do not reappear for any more positive tip voltages.

The observed tip-voltage dependence suggests that the interference pattern originates from scattering of electron waves off a tip-induced depletion region. For tip voltages below the least-invasive tip voltage $V_{\text {tip }}^{\mathrm{li}}$, the tip reduces the charge carrier density below its apex. Once the tip voltage reaches $V_{\text {tip }}<-4 \mathrm{~V}$, the Fermi energy underneath the tip is tuned into the band gap opened by the displacement field between back gate and tip. This induces an insulating depletion disk below the tip. For even larger tip voltages, this depletion disk eventually fills with a $p$-type region surrounded by a depletion ring (see [28]).

Both measurements behind the channel [cf. Figs. 1(d) and 2(a)] depict quasiperiodic interference fringes. We verify the periodicity of these patterns with high-resolution scans in the area denoted by the black rectangle in Fig. 1(d). The normalized difference of the conductance $\Delta G_{n}(x, y)$ (see [28]) measured within this area is depicted in Fig. 2(b). Here, $x(y)$ denotes the axis along the long (short) side of the rectangle in Fig. 1(d). For better visibility, each line depicted in Fig. 2(b) is slightly offset with respect to the previous line. The measurement reveals a periodicity of $\approx 29 \mathrm{~nm}$. This value is in good agreement with the Fermi wavelength estimated via a capacitive model for the van der Waals stack and assuming a circular Fermi contour. In this model, $n_{s}=\epsilon_{0} \epsilon_{r}\left[V_{\mathrm{BG}}-V_{\mathrm{BG}}^{\mathrm{CNP}}\right] /(e d)=4.12 \times 10^{11} \mathrm{~cm}^{-2}$ and therefore $\lambda_{F} / 2=28 \mathrm{~nm}$. Here, $V_{\mathrm{BG}}^{\mathrm{CNP}}$ is the experimentally observed charge neutrality point offset of the bulk regions close to the channel.

Theoretical understanding.-We interpret this jet formation in terms of the anisotropy of electron propagation in gapped BLG [35]. BLG's low-energy dispersion is anisotropic due to trigonal warping induced by interlayer skew hopping [36]. This effect is enhanced when gates open a gap, thereby inducing an asymmetry between the layers. Any anisotropy of the dispersion entails an anisotropic angular velocity distribution of adiabatic electron trajectories. To describe this anisotropy, we use the expression [36]

$$
\begin{aligned}
E^{2}= & \frac{\gamma_{1}^{2}}{2}+\frac{\Delta^{2}}{4}+\left(v^{2}+\frac{v_{3}^{2}}{2}\right) k^{2}-\left[\frac{\left(\gamma_{1}^{2}-v_{3}^{2} k^{2}\right)^{2}}{4}\right. \\
& \left.+v^{2} k^{2}\left[\gamma_{1}^{2}+\Delta^{2}+v_{3}^{2} k^{2}\right]+2 \xi \gamma_{1} v_{3} v^{2} k^{3} \cos 3 \varphi\right]^{\frac{1}{2}},
\end{aligned}
$$

for the electrons' low-energy dispersion in gapped BLG, obtained from the four-band tight-binding model. Here, $\xi= \pm 1$ indexes the two valleys $K^{ \pm}$and $\varphi=\arctan \left(k_{x} / k_{y}\right)$ is the polar angle of the momentum. We use a selfconsistent calculation for the interlayer asymmetry gap $\Delta\left(n_{e}\right)$, taking into account the electric susceptibility of the constituent monolayers and the electron density redistribution between the layers [37] induced by displacement field 
$D$ and doping (see Supplemental Material [28]). Given $D=0.143 \mathrm{~V} / \mathrm{nm}$ and the bulk charge carrier density $n_{e}=4.1 \times 10^{11} \mathrm{~cm}^{-2}$, in the single-gated region outside the channel, we find $\Delta=10.3 \mathrm{meV}$ and $E_{F}=16.0 \mathrm{meV}$ and the Fermi contour lines in the top panels of Fig. 3. We have tested the stability of the self-consistent calculation against small variations of BLG's tight-binding parameters [38] $\gamma_{1}$ (vertical interlayer coupling of the dimer sites), $v$ (in plane nearest-neighbor intralayer hopping), and $v_{3}$ (skew interlayer coupling between nondimer orbitals) in Eq. (1) and found qualitatively similar results, i.e., approximately triangular bulk Fermi contours in either valley.

For each point along the Fermi contour, the charge carrier's real space trajectory points along the direction of the normal vector as indicated in Fig. 3. The approximately triangular Fermi contours shown in Fig. 3 hence imply significant collimation of electrons in the directions orthogonal to the dispersion's flat parts (i.e., each leg of the triangle), while only few states are available in the directions of the triangle's corners. Consequently, any intravalley scattering event gives rise to three scattered

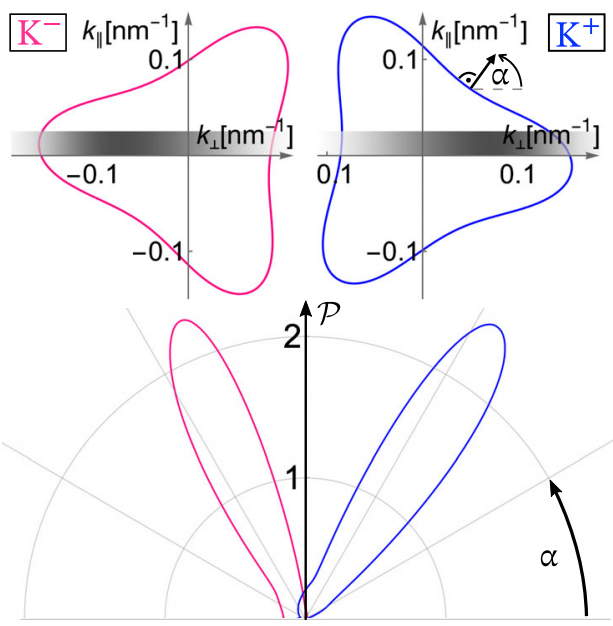

FIG. 3. Top: Fermi line for the BLG dispersion in the $K^{ \pm}$valleys in the single-gated region outside the channel for parameters $v=1.02 \times 10^{6} \mathrm{~m} / \mathrm{s}, v_{3} \approx 0.12 v$, and $\gamma_{1}=0.38 \mathrm{eV} . k_{\|}\left(k_{\perp}\right)$ is the traveling momentum parallel (perpendicular) to the channel [cf. Fig. 1(a)]. The gray bar is a density plot of the momentum distribution $\left|\Psi\left(k_{\perp}\right)\right|^{2}$ for electrons in the channel, ranging from white (no occupation) to gray (substantial occupation). We compute this distribution from the lowest channel subband state $\Psi\left(k_{\perp}\right)$ in momentum space. The charge carrier's real space trajectory points along the normal vector, prescribing the propagation direction at an angle $\alpha$. Bottom: effective angular propagation probability $\mathcal{P}$ of electrons injected from the channel, obtained by combining the angular velocity distribution originating from the bulk dispersion with the weight factors $\left|\Psi\left(k_{\perp}\right)\right|^{2}$. Two valley-polarized jets are predicted for generic orientations of the channel (here, the armchair edge of BLG is misaligned by $5^{\circ}$ with respect to the channel axis). Longer, thinner jets imply a higher probability for electrons to propagate in a well-defined direction. trajectories at mutual angles of $120^{\circ}$, whereas direct backscattering by $180^{\circ}$ requires a change of the valley [cf. Figs. 1(e) and 1(f)].

Similarly, we would expect six "jets" (three per valley) of electrons exiting the channel, if all states along the Fermi line were equally occupied. For isotropic injection from a channel at an arbitrarily oriented BLG edge, three of these jets would appear in positive propagation direction (see [28]).

The dispersion-related collimation can be further enhanced by the electrons' momentum distribution set by the internal structure of the electron subband states in the channel from which the electrons are injected. Populating different momentum states with different weights leads to a population imbalance, selectively activating the jets. We calculate the channel spectra and states using an established [6,39-41] numerical model for the BLG quantum wire (see [28]), where, for a channel conductance of $\sim 2 e^{2} / h$, only the lowest channel subbands are occupied. Hence, we weigh the occupancy of the injected states using the Fourier transform of the lowest subband channel wave function $\left|\Psi\left(k_{\perp}\right)\right|^{2}$, indicated by the gray scale bar on the $k_{\perp}$ inset in Fig. 3 .

The bottom panel of Fig. 3 shows the velocity distribution for a generic orientation of the channel (e.g., $5^{\circ}$ misalignment between the BLG zigzag axis and the sample edge) and including a $5^{\circ}$ angular broadening. Two valleypolarized electronic jets emerge from the channel at a mutual angle of $60^{\circ}$. For an arbitrary edge orientation or inhomogeneities in the channel potential, the jets may appear deformed, but the $60^{\circ}$ angle between them is a universal feature, prescribed by trigonal warping of a gapped BLG dispersion (see Supplemental Material [28]).

The anisotropic distribution and valley selectivity of the jets yields a distinct configuration of possible scattering mechanisms, the most basic of which are depicted in Figs. 1(e) and 1(f). Scattering off a smooth scattering potential, as for example introduced in the graphene layer by the tip-induced electrostatic potential, results in the valley-conserving scattering depicted in Fig. 1(e). The incoming electron (red arrow, exemplarily assumed to be in the $K^{-}$valley) scatters off the smooth scattering potential (light blue circle), resulting in three $K^{-}$-valley trajectories at a mutual angle of $120^{\circ}$ (black arrows). On the contrary, Fig. 1(f) depicts the scattering off the atomically rough sample boundary or an atomic-scale defect. The incoming trajectory (red arrow) in valley $K^{-}$can scatter off the atomic-scale scattering site (small black circle) into three valley-conserving trajectories (black arrows). However, the atomic-scale scattering site can also introduce intervalley scattering, resulting in the three additional trajectories (orange arrows) in the other valley $\left(K^{+}\right)$.

As the smooth tip potential does not allow for intervalley scattering, backscattering by $180^{\circ}$ off the tip is prohibited. This agrees with our observation that the conductance along 
the jets is generally not reduced, in contrast to the measurements of branched flow behind $\mathrm{Ga}(\mathrm{Al}) \mathrm{As}$ channels [2123], where backscattering off the tip-induced potential is the dominant mechanism. Scattering sites positioned right within the direction of the jets can give rise to secondary jets, such as the one marked by the black arrow in Fig. 1(d).

As the elastic mean free path of the charge carriers outside the channel is on the same scale as our sample size, carriers emanating from the channel predominantly follow the directions of the jets. Scattering off smooth or atomicscale scattering potentials changes the direction of the carriers away from the jets. Subsequent scattering events direct the charge carriers onto completely different paths before they reach one of the ohmic contacts, thus leading to classical conductance fluctuations. Quantum interference occurring between paths with the same valley quantum number lead to the distinct interference pattern observed along the jets in the experimental data on the scale of half the Fermi wavelength [Figs. 1(d) and 2]. Consistent with our experimental observations and in contrast to the observations in $\mathrm{Ga}(\mathrm{Al}) \mathrm{As}$ systems [21-23], the interference stripes may have any orientation with respect to the jet direction. The stripe's exact orientation is thereby determined by the individual scattering events that direct the charge carriers initially following the jets onto completely different paths before reaching an ohmic contact.

Conclusion.-We presented scanning gate microscopy measurements in close vicinity to a split-gate-defined channel in high-quality bilayer graphene. The obtained conductance maps depict two distinct jets emanating from the channel at an angle of $60^{\circ}$ with respect to each other. These jets become visible through $\lambda_{F} / 2$-periodic interference fringes that exist for tip voltages $V_{\text {tip }} \in[-10,-4] \mathrm{V}$. The magnetic field dependence of these interference patterns suggests their relation to ballistic electron transport. In contrast to similar measurements on $\mathrm{Ga}(\mathrm{Al}) \mathrm{As}$ heterostructures, the occurrence of the jets is not rooted in random electron lensing due to small-angle scattering in a correlated long-range disorder potential. Rather, the jets originate from the anisotropic band structure of bilayer graphene. Trigonal warping in conjunction with the valleydependent selection of momenta in low-energy conducting channels results in two valley-polarized jets emerging from the channel at a mutual angle of $60^{\circ}$. This observation is independent from the channel orientation with respect to the graphene edge, except for angles of $30^{\circ}$. Our observation of valley-selective electron jetting along preferred crystallographic directions presents a route to access different valley quantum numbers individually in bilayer graphene and further materials with a trigonally warped dispersion. It thus provides an important contribution to our understanding and possible utilization of the valley degree of freedom, e.g., for the initialization, transport, and readout of binary information encoded in the valley polarization and other valleytronic applications.
We thank Peter Rickhaus for fruitful discussions and Peter Märki and Thomas Bähler as well as the staff of the ETH clean room facility FIRST for their technical support. We also acknowledge financial support by the ETH Zurich Grant No. ETH-38 17-2, the Core3 European Graphene Flagship Project, and the Swiss National Science Foundation via NCCR Quantum Science and Technology. Growth of hexagonal boron nitride crystals was supported by the Elemental Strategy Initiative conducted by the MEXT, Japan, Grant No. JPMXP0112101001, JSPS KAKENHI Grants No. JP20H00354 and No. 19H05790, and the CREST (JPMJCR15F3), JST. We acknowledge funding from the Core3 European Graphene Flagship Project, the European Quantum Technology Project 2D-SIPC, the ERC Synergy Grant Hetero2D, and EPSRC Grants No. EP/S030719/1 and No. EP/N010345/1.

*cgold@phys.ethz.ch

[1] A. K. Geim and K. S. Novoselov, Nat. Mater. 6, 183 (2007).

[2] M. Yankowitz, Q. Ma, P. Jarillo-Herrero, and B. J. LeRoy, Nat. Rev. Phys. 1, 112 (2019).

[3] J. B. Oostinga, H. B. Heersche, X. Liu, A. F. Morpurgo, and L. M. K. Vandersypen, Nat. Mater. 7, 151 (2008).

[4] J. M. Pereira, P. Vasilopoulos, and F. M. Peeters, Nano Lett. 7, 946 (2007).

[5] V. Fal'ko, Nat. Phys. 3, 151 (2007).

[6] H. Overweg, H. Eggimann, X. Chen, S. Slizovskiy, M. Eich, R. Pisoni, Y. Lee, P. Rickhaus, K. Watanabe, T. Taniguchi, V. Fal'ko, T. Ihn, and K. Ensslin, Nano Lett. 18, 553 (2018).

[7] L. Banszerus, B. Frohn, T. Fabian, S. Somanchi, A. Epping, M. Müller, D. Neumaier, K. Watanabe, T. Taniguchi, F. Libisch, B. Beschoten, F. Hassler, and C. Stampfer, Phys. Rev. Lett. 124, 177701 (2020).

[8] M. Eich, F. Herman, R. Pisoni, H. Overweg, A. Kurzmann, Y. Lee, P. Rickhaus, K. Watanabe, T. Taniguchi, M. Sigrist, T. Ihn, and K. Ensslin, Phys. Rev. X 8, 031023 (2018).

[9] L. Banszerus, S. Möller, E. Icking, K. Watanabe, T. Taniguchi, C. Volk, and C. Stampfer, Nano Lett. 20, 2005 (2020).

[10] A. Rycerz, J. Tworzydło, and C. W. J. Beenakker, Nat. Phys. 3, 172 (2007).

[11] J. R. Schaibley, H. Yu, G. Clark, P. Rivera, J. S. Ross, K. L. Seyler, W. Yao, and X. Xu, Nat. Rev. Mater. 1, 16055 (2016).

[12] J. L. Garcia-Pomar, A. Cortijo, and M. Nieto-Vesperinas, Phys. Rev. Lett. 100, 236801 (2008).

[13] Z. Wang and F. Liu, ACS Nano 4, 2459 (2010).

[14] T. Stegmann and N. Szpak, 2D Mater. 6, 015024 (2018).

[15] B. A. Braem, F. M. D. Pellegrino, A. Principi, M. Röösli, C. Gold, S. Hennel, J. V. Koski, M. Berl, W. Dietsche, W. Wegscheider, M. Polini, T. Ihn, and K. Ensslin, Phys. Rev. B 98, 241304(R) (2018).

[16] K. E. Aidala, R. E. Parrott, T. Kramer, E. J. Heller, R. M. Westervelt, M. P. Hanson, and A. C. Gossard, Nat. Phys. 3, 464 (2007). 
[17] S. Bhandari, G.-H. Lee, A. Klales, K. Watanabe, T. Taniguchi, E. Heller, P. Kim, and R. M. Westervelt, Nano Lett. 16, 1690 (2016).

[18] N. Pascher, C. Rössler, T. Ihn, K. Ensslin, C. Reichl, and W. Wegscheider, Phys. Rev. X 4, 011014 (2014).

[19] B. Brun, F. Martins, S. Faniel, B. Hackens, G. Bachelier, A. Cavanna, C. Ulysse, A. Ouerghi, U. Gennser, D. Mailly, S. Huant, V. Bayot, M. Sanquer, and H. Sellier, Nat. Commun. 5, 4290 (2014).

[20] B. Brun, N. Moreau, S. Somanchi, V.-H. Nguyen, K. Watanabe, T. Taniguchi, J.-C. Charlier, C. Stampfer, and B. Hackens, Phys. Rev. B 100, 041401(R) (2019).

[21] M. A. Topinka, B. J. LeRoy, S. E. J. Shaw, E. J. Heller, R. M. Westervelt, K. D. Maranowski, and A. C. Gossard, Science 289, 2323 (2000).

[22] M. A. Topinka, B. J. LeRoy, R. M. Westervelt, S. E. J. Shaw, R. Fleischmann, E. J. Heller, K. D. Maranowski, and A. C. Gossard, Nature (London) 410, 183 (2001).

[23] M. P. Jura, M. A. Topinka, L. Urban, A. Yazdani, H. Shtrikman, L. N. Pfeiffer, K. W. West, and D. GoldhaberGordon, Nat. Phys. 3, 841 (2007).

[24] M. Koshino and E. McCann, Phys. Rev. B 80, 165409 (2009).

[25] A. Kormányos, V. Zólyomi, N. D. Drummond, P. Rakyta, G. Burkard, and V. I. Fal'ko, Phys. Rev. B 88, 045416 (2013).

[26] Y. Zhang, T.-T. Tang, C. Girit, Z. Hao, M. C. Martin, A. Zettl, M. F. Crommie, Y. R. Shen, and F. Wang, Nature (London) 459, 820 (2009).

[27] C. Gold, A. Kurzmann, K. Watanabe, T. Taniguchi, K. Ensslin, and T. Ihn, Phys. Rev. Research 2, 043380 (2020).

[28] See Supplemental Material at http://link.aps.org/ supplemental/10.1103/PhysRevLett.127.046801 for (SI) a simulation of the tip-induced potential, (SII) the sample design, (SIII) the filtering process of the experimentally measured conductance, (SIV) the magnetic field dependence of the jets, (SV) the stability of the jets against variations in displacement field and electron density, (SVI) the determination of the least-invasive tip voltage, (SVII) the normalization of the conductance, and (SVIII) the electronic structure of the channel, it includes the Refs. [29-33].

[29] M. Eich, Doctoral thesis, ETH Zurich, 2019.

[30] H. Overweg, Doctoral thesis, ETH Zurich, 2018.

[31] E. McCann and M. Koshino, Rep. Prog. Phys. 76, 056503 (2013).

[32] Y. Lee, A. Knothe, H. Overweg, M. Eich, C. Gold, A. Kurzmann, V. Klasovika, T. Taniguchi, K. Wantabe, V. Fal'ko, T. Ihn, K. Ensslin, and P. Rickhaus, Phys. Rev. Lett. 124, 126802 (2020).

[33] E. McCann and V. I. Fal'ko, Phys. Rev. Lett. 96, 086805 (2006).

[34] Whereas the upper minimum most likely corresponds to charge neutrality in bulk regions close to the channel, the lower minimum occurs due to another device not depicted in the schematic in Fig. 1(a).

[35] C. G. Péterfalvi, L. Oroszlány, C. J. Lambert, and J. Cserti, New J. Phys. 14, 063028 (2012).

[36] E. McCann, D. S. Abergel, and V. I. Fal'ko, Eur. Phys. J. Special Topics 148, 91 (2007).

[37] S. Slizovskiy, A. Garcia-Ruiz, N. Drummond, and V. I. Falko, arXiv:1912.10067.

[38] E. McCann and M. Koshino, Rep. Prog. Phys. 76, 056503 (2013).

[39] H. Overweg, A. Knothe, T. Fabian, L. Linhart, P. Rickhaus, L. Wernli, K. Watanabe, T. Taniguchi, D. Sánchez, J. Burgdörfer, F. Libisch, V. I. Fal'ko, K. Ensslin, and T. Ihn, Phys. Rev. Lett. 121, 257702 (2018).

[40] A. Knothe and V. Fal'ko, Phys. Rev. B 98, 155435 (2018).

[41] T. L. M. Lane, A. Knothe, and V. I. Fal'ko, Phys. Rev. B 100, 115427 (2019). 